\title{
MicroRNA-210 improves perfusion recovery following hindlimb ischemia via suppressing reactive oxygen species
}

\author{
JINFENG ZHANG ${ }^{1,2}$, GUOTAO RAO ${ }^{1}$, JUNYING QIU $^{1}$, RONGHUA HE $^{1}$ and QIONGTAO WANG ${ }^{1}$ \\ ${ }^{1}$ Department of Cardiology, The Central Hospital of Xiaogan, Wuhan University of Science and \\ Technology, Xiaogan, Hubei 432000; ${ }^{2}$ Department of Cardiology, Cardiovascular Research Institute, \\ Wuhan University, Hubei Key Laboratory of Cardiology, Wuhan, Hubei 430060, P.R. China
}

Received April 1, 2019; Accepted November 14, 2019

DOI: $10.3892 /$ etm.2020.9366

\begin{abstract}
In peripheral arterial disease (PAD), angiogenesis is a major process involved in repairing the microvasculature in the ischemic lower limb. MicroRNA-210 (miR-210) is a microRNA that is substantially increased in patients with PAD. However, the effects of miR-210 on angiogenesis following PAD remain elusive. In the present study, mice with hindlimb ischemia (HLI) were generated as an animal model of PAD, and miR-210 levels were overexpressed in the ischemic limb. The overexpression of miR-210 using microRNA mimics greatly improved angiogenesis and perfusion recovery; in contrast, the knockdown of miR-210 impaired perfusion recovery 28 days after HLI. Ischemic muscle tissue was harvested 7 days after experimental PAD in order to perform biochemical tests, and miR-210 antagonism resulted in increased malondialdehyde levels. In cultured endothelial cells under simulated ischemia, miR-210 mimic improved endothelial cell viability and enhanced tube formation; and a miR-210 inhibitor decreased cell survival, reduced tube formation and increased reactive oxygen species (ROS) levels. Furthermore, miR-210 antagonism increased the protein disulfide-isomerase levels in cultured endothelial cells. These results demonstrate that ischemia-induced miR-210 elevation is adaptive in PAD, and that miR-210 improves angiogenesis at least partially through decreasing ROS production.
\end{abstract}

\section{Introduction}

Peripheral arterial disease (PAD), caused by the occlusion of the arteries extending to the lower extremities, is an increasing medical problem that affects $\sim 20 \%$ of the global population $>55$ years of age (1-4). Delivery of oxygen, nutrients and other

Correspondence to: Dr Qiongtao Wang, Department of Cardiology, The Central Hospital of Xiaogan, Wuhan University of Science and Technology, 6 Guangchang Road, Xiaogan, Hubei 432000, P.R. China E-mail: wangqiongtaoxg@163.com

Key words: microRNA-210, angiogenesis, peripheral arterial disease, endothelial cells mediators to ischemic sites in patients with PAD is dependent on the growth of new blood vessels, known as angiogenesis (5-8). However, at present, there are no known medications that are available to induce functional neovascularization and thereby treat patients with PAD (9-11).

MicroRNAs (miRs) are a class of small non-coding RNAs 17-25 nucleotides in length that bind to the 3'-untranslated region (UTR) of specific mRNAs and induce mRNA degradation or suppress protein translation (12). In patients with PAD and animal models, miRs have been reported to serve important functions in tissue recovery and angiogenesis (12). Previous evidence has demonstrated that the expression of miRs are profoundly altered under hypoxic conditions; among these miRs, miR-210 is robustly upregulated following ischemia (13-15). In addition, circulating miR-210 levels have been reported to be significantly higher in patients with PAD when compared with healthy controls (16). However, whether miR-210 modulates angiogenesis in PAD remains elusive. The present study was designed to investigate the function of miR-210 in angiogenesis following PAD, and further elucidate the underlying molecular mechanisms.

\section{Materials and methods}

Murine hind limb ischemia (HLI). Unilateral HLI was generated via surgical ligation and excision of the left femoral artery in mice to create an experimental PAD model, as previously described (17). In order to understand the function of miR-210 on perfusion recovery, miR-210 was overexpressed in the ischemic limb using an intramuscular injection of $5 \mathrm{nmol} \mathrm{miR-210}$ mimic (cat. no. MC10516; Thermo Fisher Scientific, Inc., Waltham, MA, USA) 1 day prior, and 1, 3 and 5 days (4 doses) subsequent to HLI in BALB/c mice. Scrambled RNA was used as the negative control. In the present study, male BALB/c mice (aged 14-16 weeks; 20-25 g in weight, $n=18 /$ group; 6 mice were sacrificed in each group on day 7 for biochemical studies, the rest of the mice were maintained until the end of the experiments) were included and anesthetized with $3 \%$ isoflurane inhalation during the operations. Subsequent to HLI, analgesics (buprenorphine, $0.2 \mathrm{mg} / \mathrm{kg}$ ) were administered subcutaneously twice a day in the first 5 days after surgery. The health and behavior of the mice were monitored once a day. 
All procedures in the present study followed the Guide for the Care and Use of Laboratory Animals published by the US National Institutes of Health (publication no. 85-23, revised 1996). The experimental protocol was ethically approved by the Committee on Animal Experiments of Wuhan University School of Medicine (Wuhan, China). The mice were obtained from the Experimental Animal Center of Wuhan University (Wuhan, China), and were housed in a specific pathogen-free laboratory environment with ad libitum access to food and water under a 12-h light/dark cycle, and a constant humidity of $50 \pm 10 \%$ at a temperature of $25^{\circ} \mathrm{C}$. No mice succumbed to mortality during the experiment except by euthanization. For the tissue harvest, animals were euthanized by $\mathrm{CO}_{2}$ suffocation, followed by cervical dislocation 7 and 28 days after HLI.

Perfusion recovery. Mice were anesthetized and subjected to a non-invasive assessment of ischemic and non-ischemic limb perfusion using a laserDopplerperfusionimaging system(LDPI; Perimed AB, Järfälla, Sweden) at 0, 7, 14, 21 and 28 days after HLI, as previously described (18). Briefly, with this system, the perfusion was determined based on the concentration of red blood cells $x$ their velocity in the lower limb, using a beam of laser light carried by a fiber-optic probe. Perfusion of the ischemic limb was quantified and normalized to the non-surgical limb, and the results are presented as a percentage of the values in the non-ischemic side.

Immunofluorescence. Mice were sacrificed in a $\mathrm{CO}_{2}$ chamber 28 days after HLI, and fresh gastrocnemius anterior muscles from the ischemic side were imbedded in optimal cutting temperature (Tissue-Tek ${ }^{\circledR}$ O.C.T. ${ }^{\mathrm{TM}}$; Sakura Finetek USA, Inc.) compound in liquid nitrogen overnight, and then cryo-sectioned into 6- $\mu \mathrm{m}$ thick sections. The sections were blocked with 5\% goat serum (Sigma-Aldrich; Merck KGaA) for $1 \mathrm{~h}$ at room temperature, then anti-CD31 antibody (rat anti-mouse CD31; 1:100; cat. no. 550274; BD Pharmingen; BD Biosciences) was applied to acetone-fixed $\left(-20^{\circ} \mathrm{C}\right.$ for $10 \mathrm{~min}$ ) sections of gastrocnemius muscle tissue at $4^{\circ} \mathrm{C}$ overnight, followed by $1 \mathrm{~h}$ incubation at room temperature with an Alexa Fluor 555 anti-rabbit secondary antibody (1:400; cat. no. BM2004; Boster Biological Technology). Images were captured using an Olympus IX71 high-magnification microscope (Olympus Corporation), and four images were randomly selected from each sample. Capillary densities were analyzed by counting four randomly selected high-power fields (magnification, x100) and expressed as the number of $\mathrm{CD} 31^{+}$cells per field.

Biochemical assay. Malondialdehyde (MDA) in the mouse ischemic muscle homogenates was measured using a thiobarbituric acid kit (Nanjing Jiancheng Bioengineering Institute), as previously described (19). Briefly, tissue homogenates or a series of standard dilutions $(1.063,3.125,6.25,12.5,25$, 50 and $100 \mathrm{mmol} / \mathrm{l})$ were incubated with thiobarbituric acid at $95^{\circ} \mathrm{C}$ for $60 \mathrm{~min}$, and then centrifuged at 2,000 $\mathrm{x}$ g and $4^{\circ} \mathrm{C}$ for $10 \mathrm{~min}$. The supernatant was collected and extracted using butanol for spectrophotometric measurement at $532 \mathrm{~nm}$. The absolute quantity of MDA was read from a standard curve prepared from serial dilutions of the primary standard using an MDA Parameter Assay kit (R\&D Systems, Inc.).
Endothelial isolation from muscle tissue, RNA isolation and reverse transcription-quantitative polymerase chain reaction $(R T-q P C R)$ analysis. In order to quantify miR-210 levels in the endothelium, endothelial cells were isolated from the ischemic and non-ischemic hind limb muscles using CD31 antibody-bound immunobeads (cat. no. 11155D; Thermo Fisher, Scientific, Inc.) and separated using magnets. Total RNA was isolated from tissues or cells using a PureLink ${ }^{\circledR}$ RNA Mini kit (Thermo Fisher Scientific, Inc.) according to the manufacturer's protocol. A TaqMan ${ }^{\mathrm{TM}}$ MicroRNA assay for specifically miR-210 (assay ID no. 000512; Thermo Fisher Scientific, Inc.) was used for the RT-qPCR according to the manufacturer's protocol; briefly, this assay contains the primers and reagent for miR-210 reverse transcriptions, and the temperature for reverse transcription were $16^{\circ} \mathrm{C}$ for $30 \mathrm{~min}$, $42^{\circ} \mathrm{C}$ for $30 \mathrm{~min}$ and $85^{\circ} \mathrm{C}$ for $5 \mathrm{~min}$. Small nucleolar RNA U43 (assay no. 001095; Thermo Fisher Scientific, Inc.) served as an internal control for miR quantification. The amplification temperatures for RT-PCR were $95^{\circ} \mathrm{C}$ for $10 \mathrm{~min}$, followed by 40 cycles of $95^{\circ} \mathrm{C}$ for $15 \mathrm{sec}$ and $60^{\circ} \mathrm{C}$ for $1 \mathrm{~min}$. The quantification cycle $(\mathrm{Cq})$ value obtained for each gene was normalized to that of the respective internal control $(\Delta \mathrm{Cq})$. Each gene was then further normalized to the mean $\Delta \mathrm{Cq}$ value of its control group $(\Delta \Delta \mathrm{Cq})$. The final fold expression changes were calculated using the $2^{-\Delta \Delta \mathrm{Cq}}$ equation (20).

Cell culture and in vitro transfection. Human umbilical vein endothelial cells (HUVECs) were purchased from Cyagen Biosciences Inc., and grown in standard endothelial cell growth medium (Cell Applications, Inc.) with $10 \%$ fetal bovine serum (Boster Biological Technology). To mimic endothelial cells under ischemic conditions as a model for PAD, HUVECs were subjected to hypoxia (3\% oxygen; BioSpherix Medical) and serum starvation (HSS). The reason why the present study used combined HSS to mimic in vivo ischemia is that there is no oxygen or nutrition supply to the ischemic tissue without a blood supply. In vitro transfection of miRNA inhibitors or mimics at a concentration of $10 \mathrm{nM}$ was used to knockdown miR-210 expression in HUVECs, as previously described (17). Briefly, a reverse transfection protocol using neof $x$ transfection agent (Ambion; Thermo Fisher Scientific, Inc.) was used to transfect the miR-210 mimic (assay ID no. MH10516, Thermo Fisher Scientific, Inc.), miR-210 inhibitor (assay ID no. MH10516; Thermo Fisher Scientific, Inc.) or mirVana ${ }^{\mathrm{TM}}$ negative control miRNA (cat no. 4464060; Thermo Fisher Scientific, Inc.) into HUVECs for $48 \mathrm{~h}$ at $37^{\circ} \mathrm{C}$ in cell culture incubator, the concentration used for $\mathrm{miR}$ mimic or inhibitor transfection was $10 \mathrm{nM}$.

Western blotting. Subsequent to harvesting, HUVECs were homogenized in RIPA lysis buffer containing $1 \%$ protease inhibitor cocktail (Sigma-Aldrich; Merck KGaA) as previously described (19). Protein concentrations were quantified using a bicinchoninic acid protein assay kit (cat. no. PICPI23223; Thermo Fisher Scientific, Inc.). Equal amounts (30 $\mu \mathrm{g})$ of protein in homogenate samples were separated by $16.5 \%$ SDS-PAGE gel (Bio-Rad Laboratories, Inc.), transferred to nitrocellulose membranes, blocked with $5 \%$ bovine serum albumin (Sigma-Aldrich; Merck KGaA) at room temperature for $1 \mathrm{~h}$, and then blotted with primary antibodies at $4^{\circ} \mathrm{C}$ overnight 
and the corresponding peroxidase-conjugated secondary antibodies at room temperature for $1 \mathrm{~h}$. Primary antibodies against polypyrimidine tract binding protein 3 (PTBP3; cat. no. sc-100845; 1:1,000), prolyl 4-hydroxylase subunit $\beta$ (P4HB; cat. no. sc-136230; $1: 1,000)$ and $\beta$-actin (cat. no. sc-47778; $1: 5,000)$ and horseradish peroxidase (HRP) conjugated mouse anti-rabbit secondary antibodies (cat. no. sc-2357; 1:10,000) were obtained from Santa Cruz Biotechnology, Inc. The bound antibody signal was visualized using an Immun-Star HRP chemiluminescent kit (Tanon Science and Technology Co., Ltd.). The western blotting images were obtained using a Tanon 5200 Chemiluminescence Imaging System (Tanon Science and Technology Co., Ltd.). Semi-quantitative analyses of the immunoblots were performed using Image $\mathbf{J}$ software v1.8 (National Institutes of Health).

Intracellular reactive oxygen species (ROS) assay. Intracellular ROS production was detected using the cell permeating compound, 2',7'-dichlorodihydrofluorescein diacetate (DCFH-DA; Sigma-Aldrich; Merck KGaA) as previously described (19). Briefly, DCFH-DA was hydrolyzed to the fluorescent product in the presence of ROS. Following incubation with $10 \mu \mathrm{M}$ DCFHDA for $45 \mathrm{~min}$ at $37^{\circ} \mathrm{C}$, the fluorescence intensity of the HUVECs was read at $525 \mathrm{~nm}$ emission when excited at $488 \mathrm{~nm}$ in a 96 well plate reader.

Cellular viability and angiogenesis assay. For assessing cellular viability, HUVECs were seeded into a 96-well plate at a density of $1 \times 10^{4}$ cells/well ( $n=8$ per group), and then cultured under HSS conditions for $48 \mathrm{~h}$. Subsequently, the cell viability was assessed using tetrazolium dye incorporation (BioVision, Inc.) using a 96-well plate reader at a wavelength of $450 \mathrm{~nm}$ $\left(\mathrm{OD}_{450}\right)$, and the viability values were presented as a ratio to the cells transfected with the negative control. In vitro angiogenesis assays were performed as previously described (21), under HSS conditions. In brief, HUVECs were plated in 96-well plates coated with growth factor-reduced Matrigel (BD Biosciences) at a density of $1 \times 10^{4}$ cells/well, and then exposed to HSS conditions for $24 \mathrm{~h}$ to assess tube formation. This time point was selected based on the results of a previous study (17). The experiment was repeated in 6 replicates. The degree of tube formation was determined by measuring the number of loops under magnification $\mathrm{x} 40$, using a light microscope (CKX53; Olympus Corporation) using Image J software $1.15 \mathrm{~K}$ (National Institutes of Health). With four images captured at random for each well, the mean number of loops in each well were used for statistical analysis. Each experiment was repeated using at least two different batches of HUVECs.

Luciferase assay. In order to investigate the interaction between the miR-210 and its targeting site(s) on the untranslated region (3'UTR) of P4HB mRNA, $1 \mathrm{mg} / 1$ pEZX-MT06 plasmid containing P4HB 3'UTR (product ID, HmiT012109-MT06; GeneCopoeia, Inc.) or nonsense sequences (cat. no. CmiT000001-MT06; GeneCopoeia, Inc.) were transfected with $10 \mathrm{nM}$ miR-210 mimic or negative control RNA using Lipofectamine ${ }^{\circledR} 3000$ (Thermo Fisher Scientific, Inc.) and luciferase activities were measured with the Luc-Pair ${ }^{\mathrm{TM}}$ Duo-Luciferase HS Assay kit (GeneCopoeia, Inc.) according to the manufacturer's protocol, using a microplate reader (cat. no. GM3000; Promega Corporation) $48 \mathrm{~h}$ after transfection and normalized to Renilla luciferase activity.

Statistical analysis. All data were presented as the mean \pm SEM. Statistical analysis was performed with GraphPad Prism software (v7.0; GraphPad Software, Inc.). An unpaired Student's t-test was used for comparisons between two groups, and comparisons between $\geq 3$ groups were performed using a one-way analysis of variance and Tukey's post-hoc test. $\mathrm{P}<0.05$ was considered to indicate a statistically significant difference.

\section{Results}

miR-210 is upregulated in experimental PAD. Using RT-qPCR, the present study revealed that the miR-210 expression levels in the muscle from ischemic hind limbs were significantly higher compared with the non-ischemic limb 7 days after HLI $(\mathrm{P}<0.05$; Fig. 1A). The present study then sought to determine whether endothelial cells contribute to the miR-210 elevation following HLI; therefore, CD31 ${ }^{+}$cells were isolated from mice hind limbs using immunobeads, and a significantly higher miR-210 expression level was revealed compared with the non-ischemic limbs $(\mathrm{P}<0.05$; Fig. 1B) in the ischemic side. The present study then analyzed the expression of miR-210 in HUVECs under normoxic or HSS conditions for $48 \mathrm{~h}$. Consistent with what was observed in mouse muscles, miR-210 expression levels in HUVECs cultured under HSS were significantly higher compared with in HUVECs under normoxic conditions ( $\mathrm{P}<0.01$; Fig. 1C).

miR-210 mimic improves perfusion recovery and angiogenesis following HLI. In order to understand the impact of elevated miR-210 expression on perfusion recovery, the present study overexpressed miR-210 using a miRNA mimic in the left hind limb 3 days prior to HLI in BALB/c mice. The expression levels of miR-210 in the ischemic muscle were assessed 7 days subsequent to transfection, and miR-210 mimics exhibited a $\sim 90$-fold higher expression of miR-210 compared with mice receiving control RNA (data not shown). At days 14, 21 and 28 post-HLI, miR-210 overexpression resulted in significantly improved perfusion recovery compared with those receiving scrambled RNA as indicated by LDPI ( $n=10$ per group; $\mathrm{P}<0.05$; Fig. $2 \mathrm{~A}$ ). The present study then determined the capillary density in the ischemic muscle using immunostaining with CD31; mice receiving the miR-210 mimic exhibited a significantly higher capillary density compared with those receiving control RNA $(0.65 \pm 0.05$ vs. $1.79 \pm 0.08$ capillaries/fiber; $\mathrm{P}<0.01)$ in the ischemic gastrocnemius muscle 28 days subsequent to HLI (Fig. 2B).

miR-210 modulated angiogenesis in vitro. The present study next investigated the effects of miR-210 on angiogenesis in vitro using HUVECs. Transfection of an miR-210 inhibitor significantly decreased intracellular miR-210 levels compared with the negative control $(\mathrm{P}<0.01 ;$ Fig. $3 \mathrm{~A})$, and, similar to what was observed in the ischemic muscle, miR-210 antagonism in HUVECs under HSS conditions significantly decreased cell survival ( $\mathrm{P}<0.05$; Fig. 3B) and tube formation $(\mathrm{P}<0.05$; Fig. 3C) compared with the negative control. In contrast, miR-210 overexpression using miRNA mimic transfection in HUVECs 

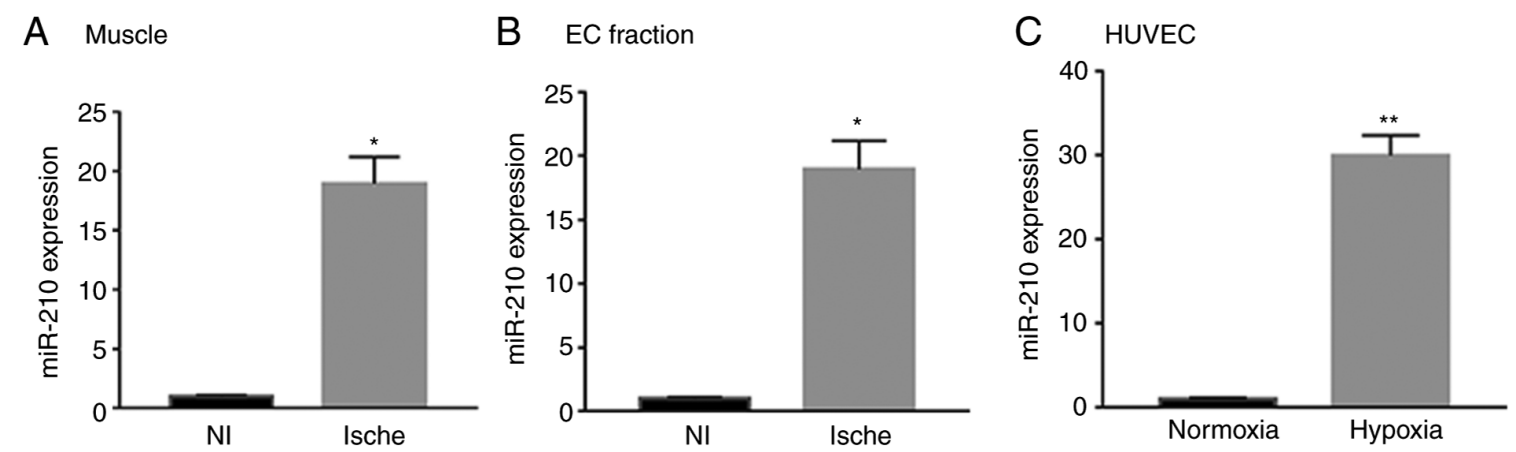

Figure 1. (A) Expression levels of miR-210 were upregulated in ischemic muscle when compared with muscle from non-ischemic limb 7 days subsequent to HLI. (B) Expression levels of miR-210 were significantly upregulated in endothelial cells isolated from ischemic muscle when compared with endothelial cells isolated from non-ischemic muscle 7 days following HLI. (C) Levels of miR-210 were higher in HUVECs cultured under hypoxic and serum-starved conditions. NI, non-ischemic muscle; Isch, ischemic muscle; HLI, hind limb ischemia. ${ }^{*} \mathrm{P}<0.05$ and ${ }^{* *} \mathrm{P}<0.01$ vs. NI/Normoxia.

A

흘 Day 0 Day 28
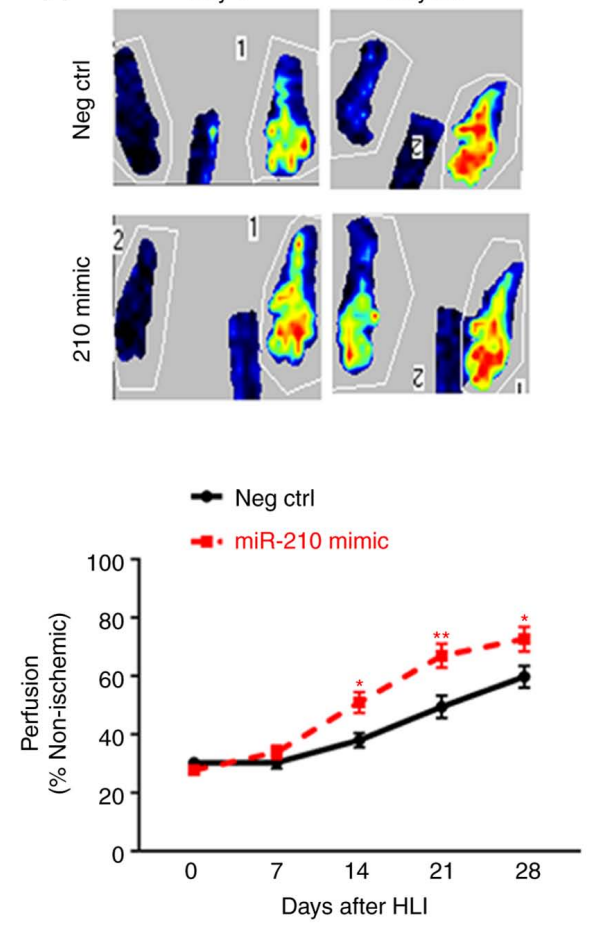

B
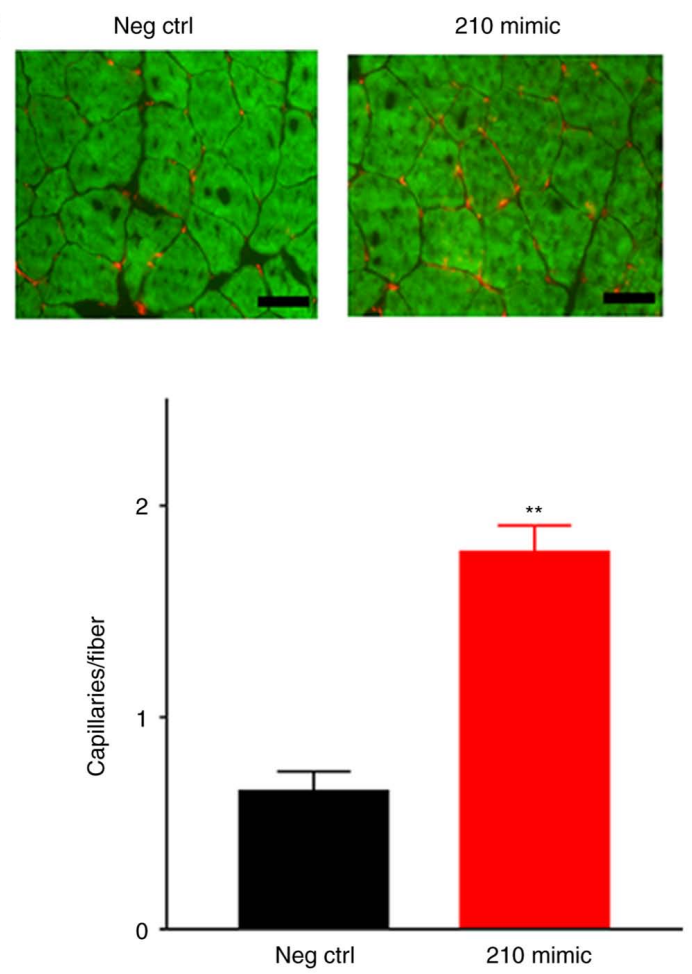

Figure 2. (A) Laser Doppler imaging demonstrated significantly increased perfusion recovery in mice treated with miR-210 mimic at day 14,21 and 28 days following HLI (n=10 per group). (B) Overexpression of miR-210 increased capillary density in ischemic hind limb muscles 28 days subsequent to HLI when compared with mice receiving negative control RNA ( $\mathrm{n}=10$ per group). Data are presented as the mean \pm standard error of the mean; scale bar, $100 \mu \mathrm{M}$. Neg Ctrl, negative control; 210 mimic, miR-210 mimic; HLI, hind limb ischemia. " $\mathrm{P}<0.05$ and ${ }^{* *} \mathrm{P}<0.01$ vs. negative control.

under HSS conditions significantly increased miR-210 expression $(\mathrm{P}<0.01$; Fig. $4 \mathrm{~A})$, in addition to cell survival $(\mathrm{P}<0.01$; Fig. 4B) and tube formation ( $\mathrm{P}<0.05$; Fig. 4C), compared with the negative control.

miR-210 increases ROS in experimental PAD. PTBP3 and $\mathrm{P} 4 \mathrm{HB}$ have previously been reported as the targets of miR-210 in ischemic tissue (13). Here, P4HB was significantly upregulated compared with the negative control $(\mathrm{P}<0.01)$, but PTBP3 was not altered in the HUVECs when miR-210 was antagonized under HSS conditions (Fig. 5A). To assess the interactions between miR-210 and P4HB mRNA, the cotransfection of miR-210 mimic and reporter plasmid with a P4HB 3'UTR region was performed and revealed to decrease P4HB expression levels, as indicated by significantly decreased luciferase activity $(\mathrm{P}<0.01$; Fig. $5 \mathrm{~B})$. $\mathrm{P} 4 \mathrm{HB}$ is a strong regulator of ROS (22), thus, the present study assessed ROS levels in HUVECs using an intracellular ROS assay. It was revealed that the miR-210 mimic significantly decreased ROS levels compared with the negative control $(\mathrm{P}<0.01$; Fig. 5C), while the miR-210 inhibitor significantly increased ROS levels compared with the negative control $(\mathrm{P}<0.01$; Fig. 5D). The present study then measured the ischemic muscle tissue levels of MDA, which have widely been used to reflect tissue ROS 

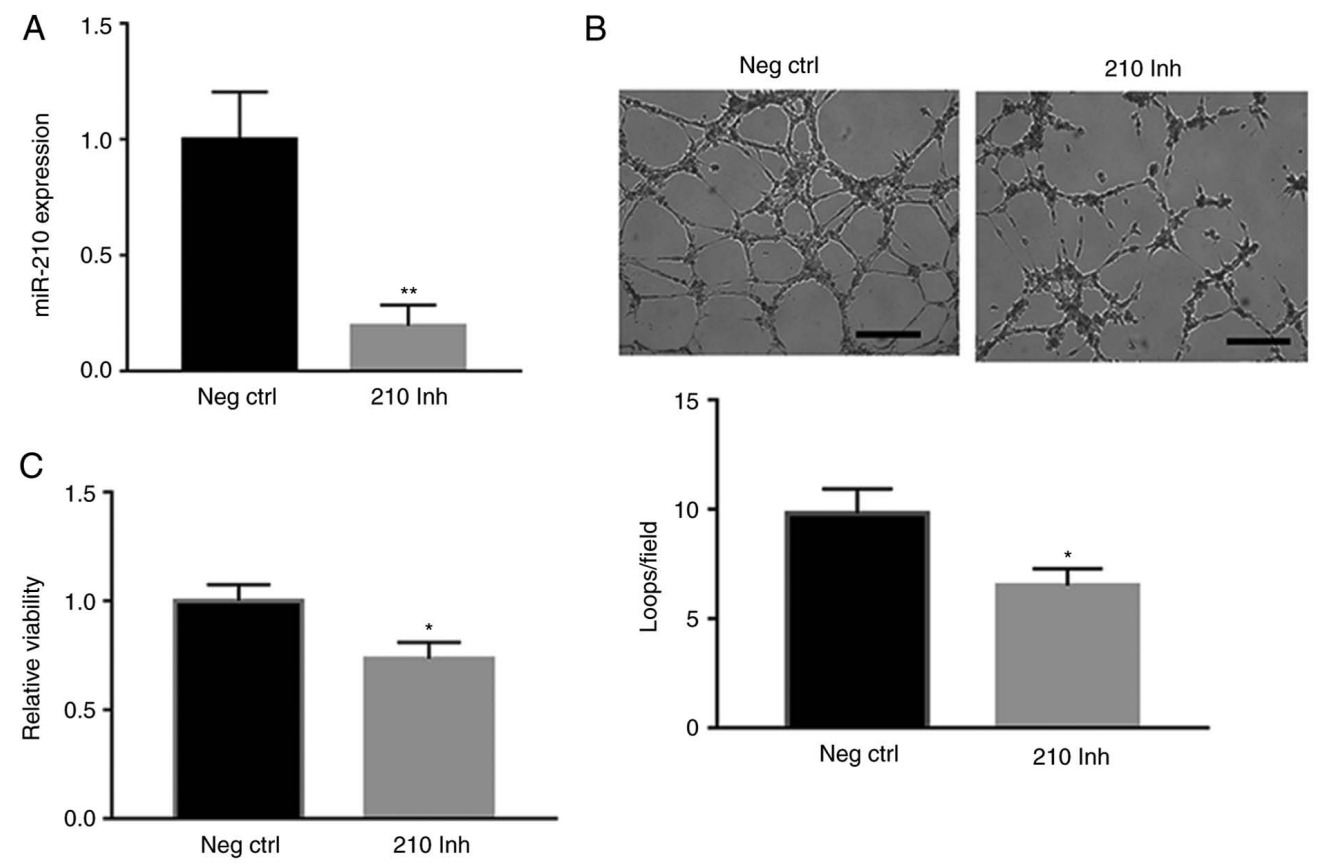

Figure 3. Transfection of miR-210 inhibitor in human umbilical vein endothelial cells significantly decreased (A) intracellular miR-210 levels, (B) tube formation and (C) cell viability. Scale bar, $100 \mu \mathrm{M}$. Data are presented as the mean \pm standard error of the mean. Neg Ctrl, negative control; 210 Inh, miR-210 inhibitor. ${ }^{*} \mathrm{P}<0.05$ and ${ }^{* * *} \mathrm{P}<0.01$ vs. negative control.

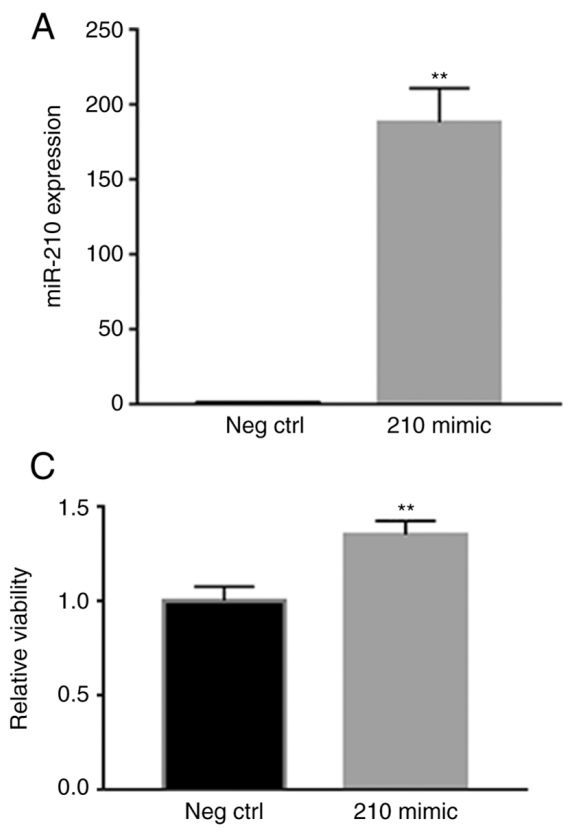

B
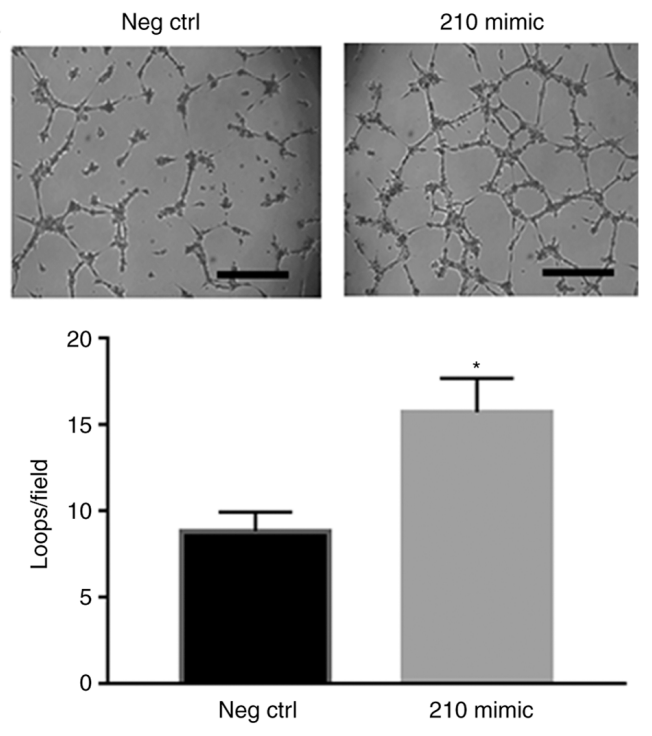

Figure 4. Transfection of miR-210 mimic in human umbilical vein endothelial cells significantly increased (A) intracellular miR-210 levels, (B) cell viability and (C) tube formation. Scale bar, $100 \mu \mathrm{M}$. Data are presented as the mean \pm standard error of the mean. Neg Ctrl, negative control; $210 \mathrm{mimic}$, miR-210 mimic. ${ }^{*} \mathrm{P}<0.05$ and ${ }^{* * *} \mathrm{P}<0.01$ vs. negative control.

bioactivity (23). miR-210 mimic significantly decreased MDA levels in the ischemic muscle 7 days following HLI compared with the negative control ( $\mathrm{P}<0.01$; Fig. 5E).

\section{Discussion}

To the best of our knowledge, the present study is the first to demonstrate that miR-210 improves angiogenesis and perfusion recovery in experimental PAD. It was also revealed that miR-210 decreased ROS levels in ischemic muscle tissue and endothelial cells, and targeted P4HB via decreasing P4HB protein levels in endothelial cells under hypoxic conditions. These results may partially explain the molecular mechanisms underlying the angiogenesis and perfusion recovery induced by miR-210.

Endothelial cells are one of the key cells involved in vascular stability and angiogenesis; however, this cell type is vulnerable 

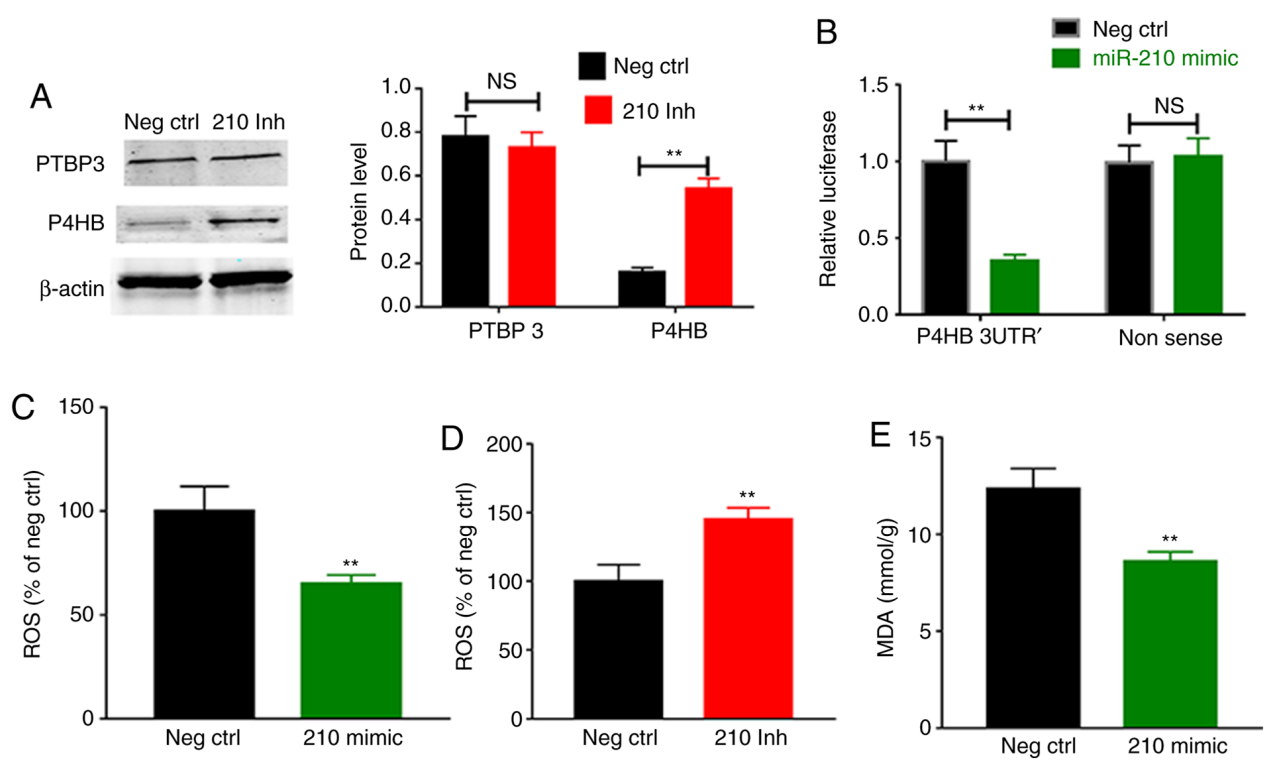

Figure 5. (A) Transfection of an miR-210 inhibitor in HUVECs did not alter the protein levels of PTBP3, but decreased P4HB protein levels. (B) Transfection of an miR-210 mimic decreased the luciferase activity in cells transfected with a P4BH 3'UTR plasmid, but not those with the negative control plasmid, which contains the luciferase sequence with a nonsense sequence in the 3'UTR region. (C) miR-210 mimic decreased the ROS levels in HUVECs cultured under hypoxia. (D) miR-210 inhibitor increased ROS levels in HUVECs. (E) Transfection of miR-210 mimic decreased MDA levels in ischemic muscle 7 days subsequent to hind limb ischemia. Scale bar, $100 \mu \mathrm{M}$. Data are presented as the mean \pm standard error of the mean. ROS, reactive oxygen species; NS, non-significant; Neg Ctrl, negative control; PTBP3, polypyrimidine tract binding protein 3; P4HB, prolyl 4-hydroxylase subunit $\beta ; 210 \mathrm{mimic}$, miR-210 mimic; 210 Inh, miR-210 inhibitor; MDA, malondialdehyde; HUVECs, human umbilical vein endothelial cells; $3^{\prime} \mathrm{UTR}$, $3^{\prime}$ untranslated region. ${ }^{* *} \mathrm{P}<0.01$ vs. negative control.

to ischemia-induced damage, ischemia-induced generation of ROS resulting in decreased synthesis and increased degradation of nitric oxide (24). In the present study, miR-210 overexpression resulted in lower ROS levels, and miR-210 knockdown resulted in higher ROS levels in ischemic muscle and cultured endothelial cells. It is of note that the HUVECs with miR-210 inhibitor transfection appeared unhealthy, which may be partially due to the miR-210 inhibition-induced endothelial cell apoptosis via ROS. A previous study reported that miR-210 modulates P4HB and PTBP protein levels and thus decreases ROS levels in acute ischemic muscles (25); in the present study, it was revealed that only $\mathrm{P} 4 \mathrm{HB}$ was regulated by miR-210 in endothelial cells. P4HB is the $\beta$-subunit of prolyl 4-hydroxylase, which functions as an endoplasmic reticulum chaperone to suppress the aggregation of misfolded proteins. A previous study in colon cancer suggested that $\mathrm{P} 4 \mathrm{HB}$ increases ROS production through the activation of nuclear factor (erythroid-derived 2)-like 2 (22). Although PTBP3 has been reported as a target of miR-210 in a previous study (25), there is no miR-210 seed sequence in PTBP3 mRNA, and thus, miR-210 regulates PTBP3 only in certain tissues, including prostate cancer and cardiovascular tissues, whereas elsewhere PTBP3 is regulated by miR-499 (26). These may be the reasons why PTBP3 is not regulated by miR-210 in endothelial cells.

A notable result of the present study is that miR-210 was higher in the ischemic limb and endothelial cells exposed to hypoxic conditions. Hypoxia-inducible factor 1- $\alpha$ (HIF-1- $\alpha$ ) is the most well-known factor that is activated under hypoxic conditions. Following activation, HIF-1- $\alpha$ translocates to the cell nucleus and binds to the promoter, activating the transcription of miR-210 upon low oxygen exposure (25). This may explain why miR-210 is upregulated in ischemic limb endothelial cells; however, this was not investigated in the present study.

In the present study, it has been demonstrated that miR-210 upregulation in endothelial cells under ischemic conditions is protective and induces angiogenesis in the ischemic tissue. However, these results are primarily based on mice studies, and results from larger animals including primates are necessary to clarify whether the results from the present study may be applied to human patients with PAD.

In conclusion, miR-210 is upregulated in endothelial cells under ischemic conditions, and this upregulation may well be adaptive as miR-210 induces angiogenesis in ischemic limb tissue, potentially via regulating oxidative stress.

\section{Acknowledgements}

Not applicable.

\section{Funding}

No funding was received.

\section{Availability of data and materials}

The datasets used and/or analyzed during the present study are available from the corresponding author on reasonable request.

\section{Authors' contributions}

$\mathrm{JZ}$ and QW conceived the project and designed the experiments. JZ, QW and RH wrote and revised the manuscript. JZ, 
QW, GR, JQ and RH performed the experiments, read and approved the final manuscript.

\section{Ethical approval and consent to participate}

The experimental animal protocol was ethically approved by the Committee on Animal Experiments of Wuhan University School of Medicine (Wuhan, China).

\section{Patient consent for publication}

Not applicable.

\section{Competing interests}

The authors declare that they have no competing interests.

\section{References}

1. Criqui MH and Aboyans V: Epidemiology of peripheral artery disease. Circ Res 116: 1509-1526, 2015.

2. Willey J, Mentias A, Vaughan-Sarrazin M, McCoy K, Rosenthal G and Girotra S: Epidemiology of lower extremity peripheral artery disease in veterans. J Vasc Surg 68: 527-535.e5, 2018.

3. Fowkes FG, Aboyans V,Fowkes FJ,McDermottMM,Sampson UK and Criqui MH: Peripheral artery disease: Epidemiology and global perspectives. Nat Rev Cardiol 14: 156-170, 2017.

4. Thiruvoipati T, Kielhorn CE and Armstrong EJ: Peripheral artery disease in patients with diabetes: Epidemiology, mechanisms, and outcomes. World J Diabetes 6: 961-969, 2015.

5. Espinola-Klein C and Savvidis S: Peripheral arterial disease. Epidemiology, symptoms and diagnosis. Internist (Berl) 50: 919-926, 2009

6. Joh JH, Joo SH and Park HC: Simultaneous hybrid revascularization for symptomatic lower extremity arterial occlusive disease. Exp Ther Med 7: 804-810, 2014.

7. Tanaka M, Taketomi K and Yonemitsu Y: Therapeutic angiogenesis: Recent and future prospects of gene therapy in peripheral artery disease. Curr Gene Ther 14: 300-308, 2014

8. Grochot-Przeczek A, Dulak J and Jozkowicz A: Therapeutic angiogenesis for revascularization in peripheral artery disease. Gene 525: 220-228, 2013.

9. Aviles RJ, Annex BH and Lederman RJ: Testing clinical therapeutic angiogenesis using basic fibroblast growth factor (FGF-2). Brit J Pharmacol 140: 637-646, 2003.

10. Owens CD and Conte MS: Medical management of peripheral arterial disease bridging the 'Gap'? Circulation 126: 1319-1321, 2012.

11. Annex BH: Therapeutic angiogenesis for critical limb ischaemia. Nat Rev Cardiol 10: 387-396, 2013.

12. Stather PW, Sylvius N, Wild JB, Choke E, Sayers RD and Bown MJ: Differential MicroRNA expression profiles in peripheral arterial disease. Circ Cardiovasc Genet 6: 490-497, 2013.

13. Guo S, Bai R, Liu W, Zhao A, Zhao Z, Wang Y, Wang Y, Zhao W and Wang W: MicroRNA-210 is upregulated by hypoxia-inducible factor- $1 \alpha$ in the stromal cells of giant cell tumors of bone. Mol Med Rep 12: 6185-6192, 2015.
14. Ikeda S, Kitadate A, Abe F, Saitoh H, Michishita Y, Hatano Y, Kawabata Y, Kitabayashi A, Teshima K, Kume M, et al: Hypoxia-inducible microRNA-210 regulates the DIMT1-IRF4 oncogenic axis in multiple myeloma. Cancer Sci 108: 641-652, 2017.

15. Pulkkinen K, Malm T, Turunen M, Koistinaho $\mathrm{J}$ and Ylä-Herttuala S: Hypoxia induces microRNA miR-210 in vitro and in vivo ephrin-A3 and neuronal pentraxin 1 are potentially regulated by miR-210. FEBS Lett 582: 2397-2401, 2008.

16. Signorelli SS, Volsi GL, Pitruzzella A, Fiore V, Mangiafico M, Vanella L, Parenti R, Rizzo M and Volti GL: Circulating miR-130a, miR-27b, and miR-210 in patients with peripheral artery disease and their potential relationship with oxidative stress. Angiology 67: 945-950, 2016.

17. Zhang J, Wang Q, Rao G, Qiu J and He R: Curcumin improves perfusion recovery in experimental peripheral arterial disease by upregulating microRNA-93 expression. Exp Ther Med 17: 798-802, 2019.

18. Albadawi H, Oklu R, Cormier NR, O'Keefe RM, Heaton JT, Kobler JB, Austen WG and Watkins MT: Hind limb ischemia-reperfusion injury in diet-induced obese mice. J Surg Res 190: 683-691, 2014.

19. Chen L, Liu C, Sun D, Wang T, Zhao L, Chen W, Yuan M, Wang J and Lu W: MicroRNA-133a impairs perfusion recovery after hindlimb ischemia in diabetic mice. Biosci Rep 38: BSR20180346, 2018.

20. Livak KJ and Schmittgen TD: Analysis of relative gene expression data using real-time quantitative PCR and the 2(-Delta Delta $\mathrm{C}(\mathrm{T}))$ method. Methods 25: 402-408, 2001.

21. Wang T, Cunningham A, Dokun AO, Hazarika S, Houston K, Chen L, Lye RJ, Spolski R, Leonard WJ and Annex BH: Loss of interleukin-21 receptor activation in hypoxic endothelial cells impairs perfusion recovery after hindlimb ischemia. Arterioscler Thromb Vasc Bio 35: 1218-1225, 2015.

22. Zhou Y, Yang J, Zhang Q, Xu Q, Lu L, Wang J and Xia W: P4HB knockdown induces human HT29 colon cancer cell apoptosis through the generation of reactive oxygen species and inactivation of STAT3 signaling. Mol Med Rep 19: 231-237, 2019.

23. Pirinccioglu AG, Gökalp D, Pirinccioglu M, Kizil G and Kizil M: Malondialdehyde (MDA) and protein carbonyl (PCO) levels as biomarkers of oxidative stress in subjects with familial hypercholesterolemia. Clin Biochem 43: 1220-1224, 2010.

24. Steven S, Daiber A, Dopheide JF, Munzel T and Espinola-Klein C: Peripheral artery disease, redox signaling, oxidative stress-Basic and clinical aspects. Redox Biol 12: 787-797, 2017.

25. Zaccagnini G, Maimone B, Di Stefano V, Fasanaro P, Greco S, Perfetti A, Capogrossi MC, Gaetano C and Martelli F: Hypoxia-induced miR-210 modulates tissue response to acute peripheral ischemia. Antioxid Redox Signal 21: 1177-1188, 2014.

26. Hosoda T, Zheng H, Cabral-da-Silva M, Sanada F, Ide-Iwata N, Ogórek B, Ferreira-Martins J, Arranto C, D'Amario D, del Monte F, et al: Human cardiac stem cell differentiation is regulated by a mircrine mechanism. Circulation 123: 1287-1296, 2011.

This work is licensed under a Creative Commons Attribution-NonCommercial-NoDerivatives 4.0 International (CC BY-NC-ND 4.0) License. 